\title{
Three-Dimensional Particle Tracking via Bifocal Imaging
}

XXXX

Vol. 0, No. 0

$\mathbf{A}-\mathbf{C}$

\author{
Erdal Toprak,,$\S$ Hamza Balci,,$\S$ Benjamin H. Blehm, ${ }^{\ddagger}$ and Paul R. Selvin ${ }^{\star, t, \neq}$ \\ Department of Biophysics, University of Illinois Urbana-Champaign, \\ Urbana, Illinois 61801, and Department of Physics, University of Illinois \\ Urbana-Champaign, Urbana, Illinois 61801
}

Received April 18, 2007; Revised Manuscript Received June 6, 2007

\begin{abstract}
We introduce a bifocal imaging method that enables three-dimensional (3D) tracking of both fluorescent and nonfluorescent particles. We accomplish this by simultaneously imaging a focused plane, for in-plane position $(x, y)$, and a defocused plane, for out-of-plane position ( $z$ ), of a molecule using a single CCD camera. We applied our method to several systems including in vivo melanosome tracking and phagocytosed fluorescent bead tracking. We have achieved 2-5 nm accuracy with a 2-50 ms time resolution.
\end{abstract}

Three-dimensional (3D) particle tracking has long been a challenge in various fields of science ranging from fluid mechanics to biology. On the other hand, the two-dimensional imaging field is significantly more mature: fluorescence imaging with one nanometer accuracy (FIONA), is able to track individual fluorophores with $1.5 \mathrm{~nm}$ accuracy; ${ }^{1}$ single molecule high-resolution colocalization (SHREC) ${ }^{2}$ and photoactivated localization microscopy (PALM) ${ }^{3}$ are able to get $<10 \mathrm{~nm}$ resolution. However, for $3 \mathrm{D}$, one needs to be able to image several micrometers away from the focal plane, particularly when imaging inside a living cell. While a number of groups have used multiplane imaging, i.e., simultaneous imaging of multiple $z$-slices, this potentially powerful technique has its shortcomings. ${ }^{4-8}$ In particular, the complicated and expensive nature of the setups, such as the necessity of multiple cameras or beam splitter mirror arrays, and the technical difficulties that accompany these complications, such as the analysis and synchronization of the data generated by these cameras, have hindered multiplane imaging from finding broad applicability.

On the other hand, defocused imaging has been a convenient way of determining the $3 \mathrm{D}$ position of a particle without the complicated setups that are typical for multiplane imaging. ${ }^{9,10}$ It is well-known that, when a particle is not in the focal plane of the imaging optics, spherical aberrations and diffraction give rise to formation of ring patterns around the particle. ${ }^{11}$ Defocused imaging uses the radius of the rings, typically the first or outermost ring, to determine the outof-plane position $(z)$, and the center of the ring to determine the in-plane coordinates $(x, y)$ of the particle. It has been

\footnotetext{
* Corresponding author. E-mail: selvin@uiuc.edu.

Department of Biophysics.

$\doteqdot$ Department of Physics.

$\S$ These authors contributed equally to this work.
}

shown that, for various sized bead particles, the radii of the rings scale linearly with the $z$-offset from the focal plane for at least several microns, which is the distance range of interest for in vivo particle tracking..$^{9,12}$ Defocused imaging is effective in determining the out-of-plane position when clear ring patterns form around the particle, i.e., more than $500 \mathrm{~nm}$ out-of-focus for $1 \mu \mathrm{m}$ sized particles. However, focused imaging is most accurate for determining the $(x, y)$ position within approximately $\pm 200 \mathrm{~nm}$ of the focal plane. Beyond this range, the loss in accuracy becomes significant. ${ }^{7}$

In this study, we present a technique that combines the advantages of multiplane imaging and defocused imaging techniques in a simple setup. We apply our technique to several biologically interesting problems, including in vivo 3D organelle and bead tracking and in vitro bead tracking. Our setup is shown in Figure 1a. A beam splitter (ms-30/ 70-1826, Optical Insights, AZ) divides the beam into two beams with a 30:70 intensity ratio, and each beam is sent to one-half of the CCD camera. Lens- 1 and Lens- 2 create space for adjusting the position of Lens-3, which shifts the focal plane of the two beams relative to each other. In the data we present, the $30 \%$ beam is focused and the $70 \%$ beam is defocused (Figure 1b). Furthermore, we have modified a commercially available "dual view cassette", part of an Optical Insights dual view apparatus (MSMI-DV-CC, Optical Insights, AZ), to combine all the optics shown in the dotted box in Figure 1a into a compact and modular single piece that can be used, or taken out, without affecting the rest of the instrument (see Figure S7 in Supporting Information for a detailed drawing of the dual view apparatus). Further details about our design and the limitations of the technique can be found in the Supporting Information notes.

To quantify the accuracy of our tracking algorithm, 200 $\mathrm{nm}$ fluorescent beads were stuck to a glass surface and 


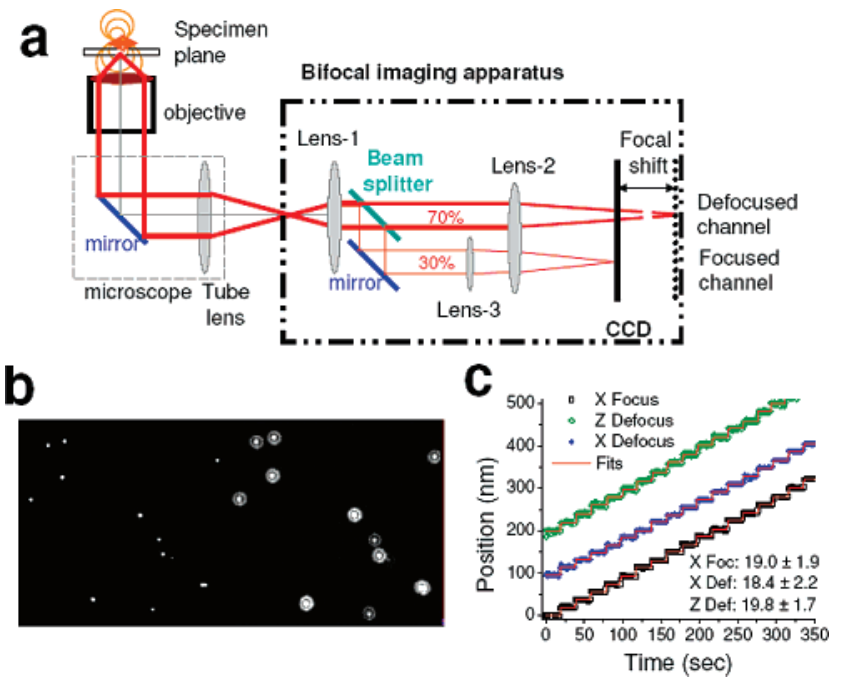

Figure 1. Schematic of the setup. (a) The focal length of Lens-3 determines the relative distance between the focal planes of the two paths. (b) A sample image of $200 \mathrm{~nm}$ fluorescent beads. The defocused image is tracked with a function of the form: $Z=P_{0}+$ $P_{1} * \exp \left[-P_{2} *\left(\left(x-x_{0}\right)^{2}+\left(y-y_{0}\right)^{2}\right)\right]+P_{3} * \exp \left[-P_{4} *\left(\left(\left(x-x_{0}\right)^{2}\right.\right.\right.$ $\left.\left.\left.+\left(y-y_{0}\right)^{2}\right)^{1 / 2}-R_{0}\right)^{2}\right]$, which is essentially a Gaussian peak surrounded by a circle of radius $R_{0}$. The magnitude of $R_{0}$ varies depending on $z$-displacement from the focal plane. The $(x, y)$ position of the particle can be determined from both $\left(x_{0}, y_{0}\right)$ parameters of the fit above, or by fitting a $2 \mathrm{D}$ Gaussian, $P_{0}+P_{1} * \exp \left[-P_{2} *(x-\right.$ $\left.\left.\left.x_{0}\right)^{2}\right] \exp \left[-P_{3} *\left(y-y_{0}\right)^{2}\right)\right]$, to the focused image. The fit to the focused image is shown to have 2 times better accuracy. (c) Sample data obtained by displacing the particle using a piezoelectric stage by $20 \mathrm{~nm}$ steps. The symbols are data for $x$-position from focused and defocused images, and $z$-position from the defocused image, and the lines are $t$-test analyzed at $P<0.05$. Tracking accuracy is $2-3 \mathrm{~nm}$ for all directions.

imaged using our image splitting apparatus. The sample was then displaced in 20,30, and $40 \mathrm{~nm}$ steps with a piezoelectric stage, and both the focused and $1 \mu \mathrm{m}$ defocused images were analyzed using our particle tracking routine (see the caption of Figure 1). These data were then fit with a $t$-test algorithm, which determines the optimal step size, as shown in Figure 1c. To compare the in-plane localization accuracy for the focused and defocused channels, the following analysis has been performed: A histogram of the difference between the data and the $t$-test steps was made for the cumulative $x$-stepping and $y$-stepping data, and this histogram was fit with a Gaussian function. The standard deviation $(\sigma)$ of this Gaussian was taken as the accuracy of localization (see Supporting Information Figure S2). For the in-focus data, $\sigma$ $=2.7 \mathrm{~nm}$, whereas for the defocused data, $\sigma=3.7 \mathrm{~nm}$ despite the focused data having only $30 \%$ of the emitted photons. Hence, assuming the localization is inversely proportional with the square root of the number of collected photons, ${ }^{13}$ the focused localization is $(3.7 / 2.7) \times(7 / 3)^{1 / 2}=$ 2.1 times better than the defocused localization for $(x, y)$ plane particle tracking.

Similar stepping analysis has been performed on 20, 40, and $500 \mathrm{~nm}$ fluorescent beads and Cyanine-3 (Cy3) fluorophores (see Supporting Information Figure S3 for data on $40 \mathrm{~nm}$ beads). In the case of $\mathrm{Cy} 3$, the short lifetime of the fluorophore makes it impossible to acquire any extended

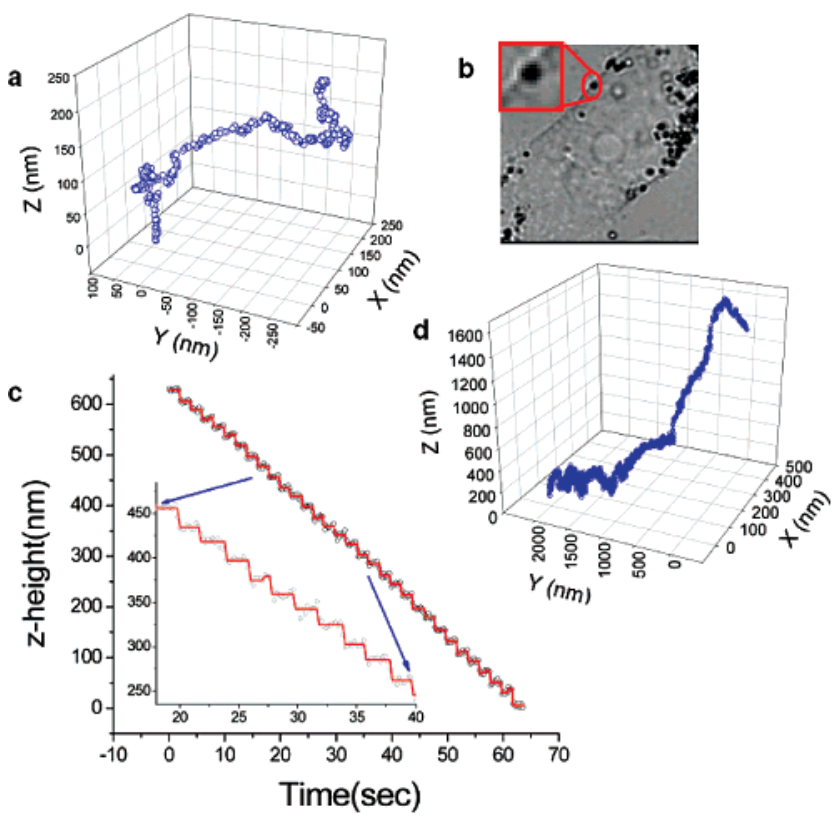

Figure 2. In vivo application of our method. (a) A sample trace of a $200 \mathrm{~nm}$ beads phagocytosed by an ARPE-19 cell shows long linear runs and abrupt direction changes in 3D. (b) An image of a melanosome treated with MSH. The inset shows a zoomed image of a melanosome with clear defocused rings. (c) Tracking a melanosome along the $z$-axis, as it is displaced with a piezoelectric stage by $20 \mathrm{~nm}$ steps, by using the rings that form around the organelle as it is defocused. The inset shows the variation of the conversion factor from ring radius to $z$-displacement for 28 different melanosomes. An important source of this variation is the size variation of the melanosomes. (d) A sample in vivo trace of a melanosome, which has the typical long linear runs and direction changes characteristic of cargo carried by multiple motors.

stepping data. Nevertheless, even along the $z$-axis, clear 40 $\mathrm{nm}$ steps were observed (see Supporting Information Figure S5). We also tried to track single quantum dots using our technique. Unfortunately, the elongated shape of quantum dots gives rise to polarization effects that made our technique inapplicable for these markers. However, an alternative method, using a cylindrical lens in the emission path to obtain the $3 \mathrm{D}$ position, has been proposed. This method is capable of tracking at 5-10 nm lateral $(x, y)$, and $30-50 \mathrm{~nm}$ axial $(z)$ resolution at typical experimental signal-to-noise ratios. ${ }^{14}$ This technique can be incorporated into our setup by replacing Lens-3 in Figure 1a with a cylindrical lens. Finally, a 3D in vitro motility assay in which kinesin coated $1 \mu \mathrm{m}$ polystyrene beads walked on inclined axonemes was designed to test our tracking algorithm. The inclined plane was formed by laying the axoneme such that one end would be on a polymer step and the other on the glass surface. The trajectory of the kinesin coated bead which moved on this track successfully reproduced the inclined plane shape of the axoneme, confirming the capability of defocused imaging to track $1 \mu \mathrm{m}$ beads in vitro in 3D. For trajectories, pictures, and movies regarding these experiments, see the Supporting Information notes.

Figure 2 shows two in vivo applications of our method. In the first case, $500 \mathrm{~nm}$ fluorescent beads (Molecular Probes, F-8812) were phagocytosed into ARPE-19 cells and imaged 
with a CCD camera at $50 \mathrm{~ms}$ time resolution. Figure 2a shows a trace of one of these beads as it is transported within the cell. The bead moves on linear tracks for a few hundred nanometers and abruptly switches orientation, presumably moving to a new track, and moves for another few hundred nanometers. How different kinds of motors, i.e., kinesin, dynein, and myosin, are regulated while acting on the same cargo is an important problem in intracellular transport. Run lengths and frequency of directional changes are two of the important parameters that have been studied in $2 \mathrm{D} .{ }^{15}$ Figure 2a shows that our imaging technique can reliably address such problems in 3D.

The second application in Figure 2 is on melanosomes, nonfluorescent organelles, which carry the pigment melanin in melanophore cells. Figure $2 \mathrm{~b}$ shows a melanophore after it was treated with melanocyte stimulating hormone (MSH) in order to disperse the melanosomes to the cell periphery and hence enable tracking of individual organelles. The black particles in the image are melanosomes. The inset of Figure $2 \mathrm{~b}$ shows a defocused image of a melanosome. To test the capability of defocused imaging to track the $z$-position of melanosomes, a stepping analysis similar to that of Figure $1 \mathrm{c}$ was performed on surface stuck melanosomes in vitro. The average step size of $20.1 \pm 3.2 \mathrm{~nm}$ for the 31 steps shown in Figure 2c is in good agreement with the $20 \mathrm{~nm}$ piezoelectric stage steps. The bottom inset of Figure 2c shows a zoomed view of the stepping behavior. Figure $2 \mathrm{~d}$ shows an in vivo track of a melanosome imaged at $2.2 \mathrm{~ms}$ time resolution. Long linear runs and abrupt direction changes are clearly visible, suggesting that our method is capable of tracking melanosomes in vivo. For more data, including a movie of the trace in Figure $2 \mathrm{~d}$ and a statistical analysis of stepping behavior on 28 different melanosomes, see the Supporting Information notes.

In conclusion, we propose a simple 3D particle tracking method and show its applicability for in vivo and in vitro systems using both fluorescent and nonfluorescent particles. The advantages of our technique are its simplicity and modularity in the sense that it can easily be integrated into existing optical setups without sophisticated engineering that requires multiple cameras or complicated optics. The particle tracking is also very simple because the data from both planes are simultaneously read via the same CCD camera.

Acknowledgment. We thank Sheyum Syed, Taner Ozel, Jeff Reifenberger, and David Posson for their help and advice. We thank Karl Garsha and Chris Baumann for providing us a detailed drawing of the dual view apparatus (Figure S7, Supporting Information). This work was supported by NIH grants AR44420 and GM68625.

Supporting Information Available: Typical calibration analysis for converting the ring radius of a defocused image to $z$-position for $200 \mathrm{~nm}$ fluorescent bead; comparison of focused vs defocused tracking accuracy for in-plane $(x-y$ plane) particle motility; two examples of the capability of defocused analysis; polystyrene beads of $1 \mu \mathrm{m}$ diameter; trace of a single $\mathrm{Cy}-3$ displaced with a piezoelectric stage with $40 \mathrm{~nm}$ steps; image of the Arpe19 cells with phagocytosed $200 \mathrm{~nm}$ fluorescent beads excited with a $532 \mathrm{~nm}$ wavelength laser; detailed drawing of the dual view apparatus and the modifications for application. Movies of the experiment (AVI). This material is available free of charge via the Internet at http://pubs.acs.org.

\section{References}

(1) Yildiz, A.; Forkey, J. N.; McKinney, S. A.; Ha, T.; Goldman, Y. E.; Selvin, P. R. Science 2003, 300, 2061-2065.

(2) Churchman, L. S.; Okten, Z.; Rock, R. S.; Dawson, J. F.; Spudich, J. A. Proc. Natl. Acad. Sci. U.S.A. 2005, 102, 1419-1423.

(3) Betzig, E.; Patterson, G. H.; Sougrat, R.; Lindwasser, O. W.; Olenych, S.; Bonifacino, J. S.; Davidson, M. W.; Lippincott-Schwartz, J.; Hess, H. F. Science 2006, 313, 1642-1645.

(4) Willert, C. E.; Gharib, M. Exp. Fluids 1992, 12, 353-358.

(5) Nguyen, L. K.; Bravo-Zanoguera, M. E.; Kellner, A. L.; Price, J. H. Proc. SPIE 2000, 3921, 31-40.

(6) Prabhat, P.; Ram, S.; Ward, E. S.; Ober, R. J. Proc. SPIE 2006, 6090, 60900L-1.

(7) Ram, S.; Chao, J.; Prabhat, P.; Ward, E. S.; Ober, R. J. Proc. SPIE 2007, 6443, 64430D-1

(8) Ram, S.; Ward, E. S.; Ober, R. J. Proc. Natl. Acad. Sci. U.S.A. 2006 , $103,4457$.

(9) Speidel, M.; Jonas, A.; Florin, E. L. Opt. Lett. 2003, 28, 69-71.

(10) Wu, M.; Roberts, J. W.; Kim, S.; Koch, D. L.; DeLisa, M. P. Appl. Environ. Microbiol. 2006, 72, 4987-4994.

(11) Born, M.; Wolf, E., Principles of Optics, 8th ed.; Pergamon: New York, 1959.

(12) Wu, M.; Roberts, J. W.; Buckley, M. Exp. Fluids 2005, 38, 461465.

(13) Thompson, R. E.; Larson, D. R.; Webb, W. W. Biophys. J. 2002, $82,2775-2783$.

(14) Holtzer, L.; Meckel, T.; Schmidt, T. Appl. Phys. Lett. 2007, 90, 053902.

(15) Gross, S. P. Phys. Biol. 2004, 1, R1-R11.

NL0709120 